\title{
UNCERTAINTY FOR BURNT AREA PRODUCTS
}

\author{
J Brennan ${ }^{1,2}$, J Gómez-Dans ${ }^{1,2}$, P Lewis ${ }^{1,2}$, M Chernetskiy $^{2}$, A Heil $^{3}$ \\ ${ }^{1}$ National Centre for Earth Observation (NCEO), UK \\ ${ }^{2}$ Dept. of Geography, University College London, UK \\ ${ }^{3}$ Dept. of Atmospheric Chemistry, Max Plack Institute for Chemistry, Germany
}

\begin{abstract}
Burnt area (BA) products are usually provided as a binary mask, indicating whether within a particular time interval, a pixel has or has not burnt. However, this is an inference derived from assessing e.g. the change in reflectance due to the fire. These calculations are prone to uncertainty from a number of sources: thermal noise in the sensor, residual atmospheric correction shortcomings or insufficient temporal sampling, etc. In this contribution, we aim to provide a framework for uncertainty characterisation of BA products. The uncertainty framework is Bayesian in nature, and provides a way to propagate uncertainty from the observations, across scales, but also allows one to propagate uncertainty in algorithm parameterisation. We illustrate the framework with a simple example based on logistic regression. Finally, we discuss how the uncertainty at the pixel level can be aggregated to the climate modeller grid (CMG), providing a consistent way to treat uncertainty from the observations and algorithm parameters to the final products.
\end{abstract}

Index Terms - Uncertainty, Burnt Area, ECV

\section{INTRODUCTION}

The generation of consistent terrestrial remote sensing products with associated uncertainties is necessary for the monitoring of the Earth System. The Global Climate Observing System (GCOS) have outlined 54 observable parameters as essential climate variables (ECVs) which are crucial to the characterisation an long-term monitoring of the climate system $[1,2]$. Burnt Area (BA) forms an integral part of the Fire Disturbance ECV. The only possible way to monitor Burnt Area globally over the past three decades is from the processing of remote sensing measurements. The inference of fire occurrence at both the pixel (10s-100s metres) scale and Climate Model Grid (CMG, $0.05-0.25^{\circ}$ ) requires the processing of these measurement via Burnt Area algorithms. This task, like any in remote sensing is an inverse problem subject to uncertainty from measurement errors and uncertainties within the retrieval algorithm [3, 4]. We therefore argue that there is a considerable necessity for uncertainty quantified BA products for the production of BA ECVs.

\section{UNCERTAINTY PROPAGATION IN BA ALGORITHMS}

A burnt area algorithm $\mathbf{F}$ provides a retrieval of whether the landsurface has burnt or not $B$ given observations $d$ and the properties of the model $w$ :

$$
B=\mathbf{F}(d, w)
$$

Within remote sensing there has been a move towards a recognition of the uncertainties in observations and retrieval algorithms [5]. As such we propose an uncertainty framework suitable for burnt area detection. Fundamentally at the pixel level the uncertainty in the retrieval can be characterised probabilistically. We define the probability that the pixel has burnt as $p_{b}$. Within a Bayesian framework the conditional dependencies between the algorithm retrieval and observations can be represented by conditional probability distributions. Such that the retrieval problem can be phrased as:

$$
P(B \mid w, d)=\frac{P(d \mid w) P(w)}{P(d)}
$$

To demonstrate this we consider a simple illustrative example (see figure 1). An algorithm is trained to select a small training dataset $\mathcal{T}$ of burned and unburned pixels for values of $d \mathrm{NBR}$.

$$
\mathcal{T}=\{X, y\}
$$

Where $y$ indicates whether the pixel is a priori known to be burned or unburned and $X$ are a vector of some suitable metric that is known to be sensitive to fire (for example, a vegetation index like dNBR, but not limited to vegetation indices):

$$
X=\left[x_{i}, \ldots, x_{N}\right]^{T}
$$

The uncertainty in the observations $X$ are assumed to be normally distributed. The distribution of possible values for $X$ is then described by the covariance matrix $\Sigma$. For this purpose we will assume the observations are independent, each with a unique standard deviation such that $\Sigma$ resembles: 


$$
\Sigma=\left(\begin{array}{ccccc}
\sigma_{i}^{2} & & & & \\
& \sigma_{i}^{2} & & & \\
& & \ddots & & \\
& & & \sigma_{i}^{2} & \\
& & & & \sigma_{N}^{2}
\end{array}\right)
$$

Here, we choose to formulate the BA algorithm as a logistic regression problem, although any other approach could be used instead. Logistic regression provides a technique for learning a discriminative model for the classification $P(B \mid x)$. The general form of a logistic regression is:

$$
P(B \mid x, w)=\operatorname{Ber}\left(B \mid g\left(w^{T} x\right)\right)
$$

where $g()$ is the sigmoid function:

$$
P=g\left(w^{T} x\right)=\frac{1}{1+e^{-w^{T} x}}
$$

A logistic regression classifier represents a suitably generic template for a probabilistic BA algorithm. Primarily most present BA algorithms are solved as an explicit two class classification problem in which information about the class conditional distributions of burnt and unburnt pixels are described and used to evaluate a new observation. Similarly many present BA algorithms involve discriminative classifiers in which the decision boundary between the burnt and unburnt classes is determined either by a priori expectation or via a training methodology. Further logistic classifiers have been used in many burned area detection studies $[6,7,8]$. As outlined above the primary step involves training the parameters of the model $w$ from a training dataset $\mathcal{T}$. Given the training dataset the log posterior of $P(w \mid X, y)$ is:

$$
\begin{aligned}
\log P(w \mid X, y) & =\sum_{i} y_{i} \log \left(g\left(w^{T} x_{i}\right)\right) \\
& +\left(1-y_{i}\right) \log \left(1-g\left(w^{T} x_{i}\right)\right) \\
& +\log \left(\left|x-x_{0}\right|^{T} \mathbf{C}_{\text {obs }}^{-1}\left|x-x_{0}\right|\right)
\end{aligned}
$$

To propagate the uncertainty in the observations $X$, we draw samples from $P(w \mid X, y)$ via an MCMC sampler [9]. We then wish to predict on an pixel $p$ whether it is burnt or unburnt with the model. The observation of dNBR in the pixel is described by a normal distribution with an uncertainty specified by $\mathrm{dNBR}_{\sigma}$ which for brevity we call the random variable $x$. The posterior is therefore at the pixel scale defined by:

$$
P(B \mid, w, x, y)=\frac{P(w \mid X, y) P(w)}{P(x)}
$$

To provide a final prediction on whether the pixel is burned or not we need to marginalise over the uncertainty in the observations and model. This provides the predictive posterior, $p_{b}$ :

$$
p_{b}=\int_{w} \int_{x} P(B \mid, w, x, y)
$$

So $p_{b}$ encompasses how certain one is about the pixel being burned, and it includes the uncertainty in the observations, the magnitude of the burn signal, the availability of observations at the time of the observation, etc. $p_{b}=0$ is absolute certainty in the pixel not being burned, and $p_{b}=1$ is absolute certainty of the pixel being burned. Figure 1 shows an illustrative example with real data.

\section{AGGREGATION TO COARSE SCALE}

Climate users of Burnt Area products typically prefer products at resolutions equivalent to climate models. This requires a coarse scale representation of the Burnt Area as the area burned per e.g. half-degree grid cell. Typically, this has been achieved by adding up all the pixels that have been classified as being burned. However, this approach does not account for uncertainty in each pixel being detected as burned or not. In this Section, we investigate how to aggregate per pixel information provided by $p_{b}$ to coarser scales.Under the assumption that each pixel is independent, and assuming that each pixel can either be burned or unburned with a probability $p_{b}$, we have a combination of $N$ Bernoulli trials. The distribution of this aggregate is governed by a Poisson Binomial distribution. While calculating the actual distribution from the set of $N$ samples is computationally complex, the mean and standard deviation are quite simple:

$$
\begin{aligned}
\mu & =\sum_{i=1}^{N} p_{b}^{(i)} \\
\sigma & =\sum_{i=1}^{N} p_{b}^{(i)}\left(1-p_{b}^{(i)}\right)
\end{aligned}
$$

These two expressions are interesting. The mean burned area is equal to the sum of burned pixels if the burned pixels are assumed to have $p_{b}=1$ and $p_{b}=0$ is assumed to hold for unburned pixels. In this case, the variance of the distribution is also zero. A more realistic scenario will happen if $0<p_{b}<1$, although one still expects that a good algorithm will have very high $p_{b}$ for detected pixels and a very low $p_{b}$ for unburned pixels. Further, note that the Poisson binomial is a discrete distribution, but if $N$ is high, it can be approximated by a normal distribution in most cases. We illustrate this with a simple synthetic experiment. The spatial distribution of $p_{b}$ is shown in Fig. 2. Some burnscars can be seen in yellow colour, but the "speckliness" means that not all pixels are clearly detected. A standard BA algorithm will apply some threshold to decide what pixels are burned and which pixels are not burned. Adding them up results in the vertical line shown in Fig. 3, with a variance of 0 (no uncertainty). 

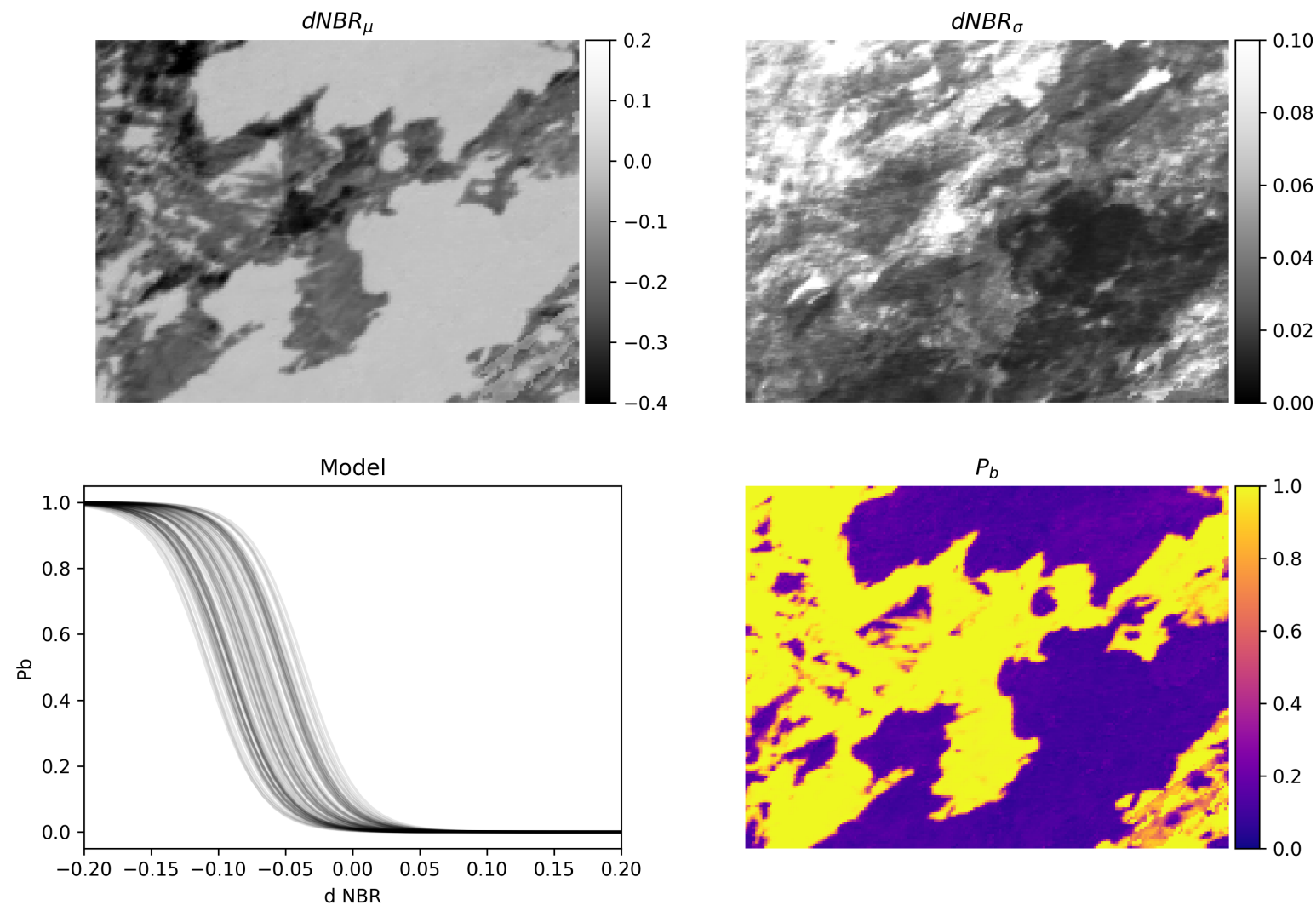

Fig. 1. Example of a fully uncertainty quantified BA algorithm on real data. Top left) dNBR over a burn scar. Top right) uncertainty in dNBR. Bottom left) Draws from the logistic classification model. Bottom right) posterior predictive estimate of burning $p_{b}$.

Using the proposed method, one gets the probability density function (pdf) shown in Fig. 3, where both the Poisson Binomial version and the normal approximation are shown. We note that in this case, the total number of burned pixels was 2700 , and we see how the proposed method actually encompasses this value within it's main lobe. This approach to aggregation is both pragmatic and clear. The main issues with it are that it relies on $p_{b}$ being credible, and it makes a strong assumption of independence of $p_{b}$, which will not be the case for algorithms that e.g. do spatial region growing processing. The first shortcoming is addressed in the rest of this contribution.

\section{RESULTS}

The framework introduced provides a probabilistic formulation for fully uncertainty quantified burnt area products across spatial scales. The framework is demonstrated with a simple BA algorithm on real and synthetic data to illustrate how the per-pixel probability of burn $p_{b}$ is evaluated. This so-called $p_{b}$ can then be aggregated using the scheme presented in the last section to climate grids.

\section{REFERENCES}

[1] Stephan Bojinski, Michel Verstraete, Thomas C Peterson, Carolin Richter, Adrian Simmons, and Michael Zemp, "The concept of essential climate variables in support of climate research, applications, and policy," Bulletin of the American Meteorological Society, vol. 95, no. 9, pp. 1431-1443, 2014.

[2] Rainer Hollmann, Chris J Merchant, Roger Saunders, Catherine Downy, Michael Buchwitz, Anny Cazenave, Emilio Chuvieco, Pierre Defourny, Gerrit de Leeuw, René Forsberg, et al., "The esa climate change initiative: Satellite data records for essential climate variables," Bulletin of the American Meteorological Society, vol. 94, no. 10, pp. 1541-1552, 2013.

[3] Sean Twomey, Introduction to the mathematics of inversion in remote sensing and indirect measurements, vol. 3 , Elsevier, 2013.

[4] Clive D Rodgers, Inverse methods for atmospheric 


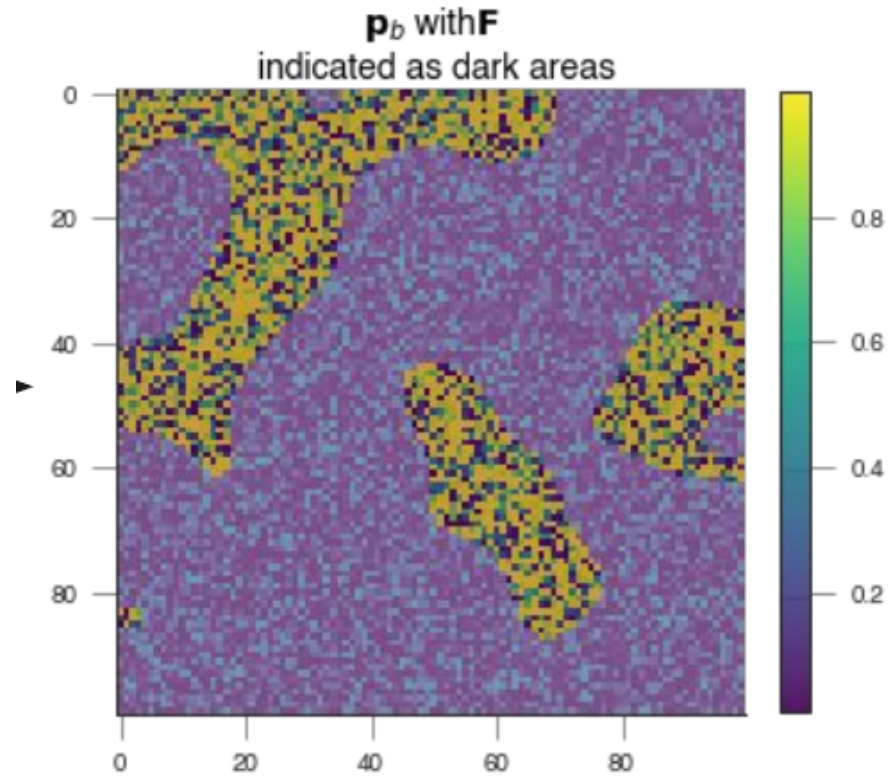

Fig. 2. Spatial distribution of $p_{b}$ over a synthetic fire

sounding: theory and practice, vol. 2, World scientific, 2000.

[5] AC Povey and RG Grainger, "Known and unknown unknowns: uncertainty estimation in satellite remote sensing," Atmospheric Measurement Techniques, vol. 8, no. 11, pp. 4699-4718, 2015.

[6] N Koutsias and M Karteris, "Burned area mapping using logistic regression modeling of a single post-fire landsat5 thematic mapper image," International Journal of Remote Sensing, vol. 21, no. 4, pp. 673-687, 2000.

[7] RH Fraser, R Fernandes, and R Latifovic, "Multitemporal mapping of burned forest over canada using satellite-based change metrics," Geocarto International, vol. 18, no. 2, pp. 37-47, 2003.

[8] Aitor Bastarrika, Emilio Chuvieco, and M Pilar Martín, "Mapping burned areas from landsat tm/etm+ data with a two-phase algorithm: Balancing omission and commission errors," Remote Sensing of Environment, vol. 115, no. 4, pp. 1003-1012, 2011.

[9] D. Foreman-Mackey, D. W. Hogg, D. Lang, and J. Goodman, "emcee: The MCMC Hammer,", vol. 125, pp. 306, Mar. 2013.

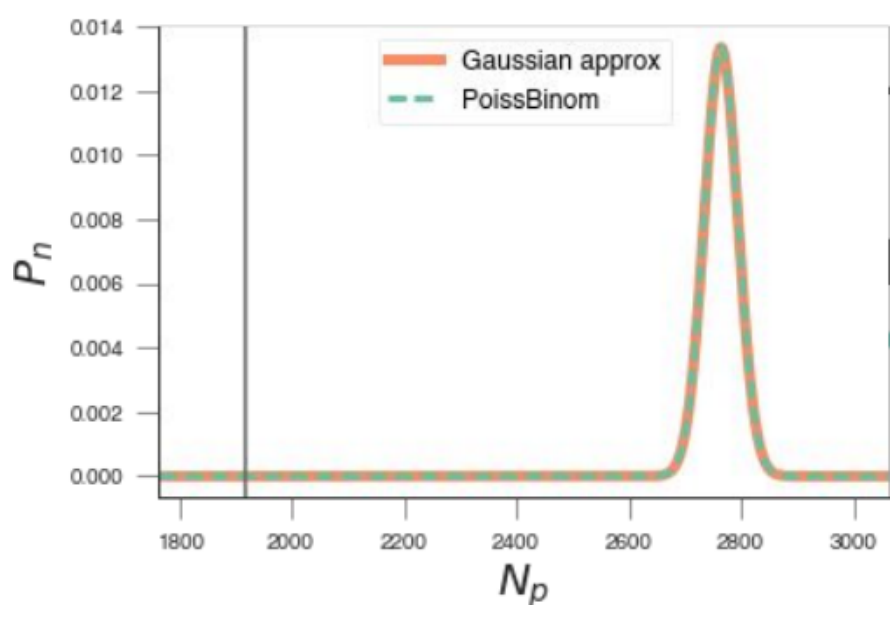

Fig. 3. Aggregating the data from Fig. 2 to a corarse resolution grid. The black vertical line shows the number of burned pixels obtained adding all the pixels where $p_{b}>0.5$ (an arbitrary threshold). The green and orange curves are the distribution of burned pixels in the aggregated cells using (respectively) the Poisson binomial pdf and the Normal approximation. The true value of burned pixels was 2700 . 